\title{
Construction and Characterisation of Double Layer Capacitors
}

\author{
Z. Stevićc ${ }^{a, *}$, M. RajČić-Vujasinovićc ${ }^{a}$, S. Bugarinović ${ }^{b}$ And A. Dekanski ${ }^{c}$ \\ ${ }^{a}$ University of Belgrade, Technical Faculty in Bor, Serbia \\ ${ }^{b}$ Scholar Ministry of Science and Technological Development, Serbia \\ ${ }^{c}$ Institute of Chemistry, Technology and Metallurgy, Belgrade, Serbia
}

\begin{abstract}
Electrochemical double-layer capacitor (also called supercapacitor) is an electrochemical energy storage device with a high power density, which could be used in application such as pulse power devices or electric vehicles. The paper describes construction of an electrochemical double-layer capacitor that consists of two electrodes immersed in an electrolyte with a separator between them. One electrode consists of a current collector in contact with the active material, while the other one is a counter electrode. Commercially available activated carbon (AC) material, composite $\mathrm{RuO}_{2}+\mathrm{AC}$ and natural copper sulfides are used as the active electrode material. Characterization has been performed by an electrochemical system fully developed at Technical Faculty in Bor. The system is based on a PC P4, a commercially available ADDA converter and an external interface for analog signal processing. The software platform is LabVIEW 8.2 package and application software is adapted to investigations of the systems containing high capacitances.
\end{abstract}

PACS numbers: 82.47.Uv, 82.45.Fk, 82.80.Fk, 01.50.hv, 01.50.Kw, 01.50.Lc, 01.50.Pa

\section{Introduction}

Supercapacitors fulfill a very wide area between accumulator batteries and conventional capacitors taking into account specific energy and specific power. Batteries and fuel cells are typical devices of small specific power, while conventional capacitors can have specific power higher than $1 \mathrm{MW} / \mathrm{dm}^{3}$, but they suffer from low power density and low cyclability (usually $<1000)[1-5]$. Their specific energy capacity is limited by the breakdown field $\left(\mathrm{V} \mu \mathrm{m}^{-1}\right)$ of their dielectric material. The electrochemical double-layer capacitor (EDLC) has been developed for these cases which need a large energy density $\left(\mathrm{Wh} \mathrm{kg}^{-1}\right)$, high power density $\left(\mathrm{W} \mathrm{kg}^{-1}\right)$, and long cycle life $(>100000)$. The EDLC is a new type of capacitor offering new features intermediate between those of a battery and a conventional capacitor.

Considering applying, there are four groups of supercapacitors: (1) capacitors for supplying electronic consumers, (2) filter capacitors, (3) power electronic capacitors, and (4) electric haulage capacitors. Depending on applying place, different characteristics of supercapacitors can be more or less taken into account. Some of them are of crucial importance for capacitor choice, and some of them can be of no importance at all.

First group includes capacitors for supplying electronic consumers of small power and very low voltage (CMOS

\footnotetext{
* corresponding author; e-mail: zstevic@tf.bor.ac.rs
}

memories, watches, micro controllers, intelligent sensors, etc.). They are most frequently produced as a miniature cell of great capacitance. Crucial role at choice have cell voltage, capacitance and self-discharging current. Inner (serial) resistance is resistance of less importance.

Second group includes filter capacitors for obtaining "ideally" filtered DC voltages. Apart from great capacitance, cell voltage is of great importance for them, and for achieving working voltage, it most frequently requires serial connection of several cells. Self-discharging current and serial resistance are practically of no importance.

Third group of supercapacitors in future will have great application in power electronics complexes of medium power, using for electrical energy reservoirs in transient regime. There is an actual possibility to replace soon massive inductivities, which are at the same time great sources of electromagnetic disturbances. Used for those purposes, supercapacitor must have great capacitance and relatively great working voltage (implicating serial cell connection and all problems related to that). Inner resistance has to be rather small, and leakage current is not of greater importance.

Most strict requirements are related to capacitors of the fourth group, applied in electric haulage, i.e. for vehicles of the future. Nowadays, batteries of several hundreds farad capacitance with working voltage of several hundred volts have been produced. Beside great capacitance and relatively high working voltage, these capacitors must have great specific energy and power (because of limited space in vehicle). Considering their specific 
power, they have great advantage in relation to accumulator batteries, but, on the other side, they are incomparably weaker considering specific energy.

Hence, ideal combination is parallel connection of accumulator and condenser batteries (Fig. 1). In an established regime (normal drawing) vehicle engine is supplied from accu-battery, and in the case of rapidly speeding, from supercapacitor. A very important is the fact that in the case of abrupt breaking, complete mechanical energy could be taken back to system by converting into electrical energy only in the presence of supercapacitor with great specific power. Because of mentioned reasons, inner resistance of these supercapacitors has to be extremely small. Leakage current is not of essential importance. Vehicles with such drive are not still in wide use, and the reasons for that are for sure economic.

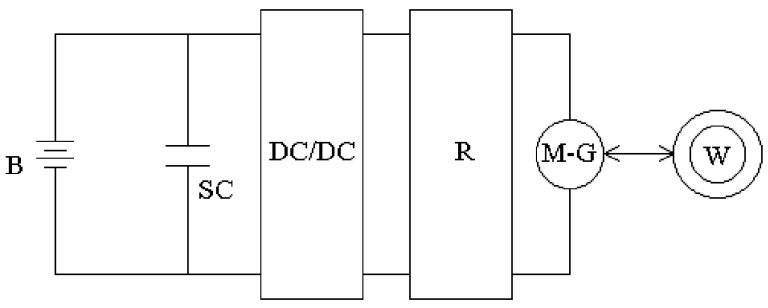

Fig. 1. Scheme of electrical drive vehicle with supercapacitor, with possibility for using the breaking energy, $\mathrm{B}$ - DC voltage source; SC - supercapacitor; DC/DC - one-way voltage converter; $\mathrm{R}$ - regulator; $\mathrm{M}-\mathrm{G}$ engine-generator (depending on working regime; $\mathrm{W}-$ drive wheels).

Critical component at each hybrid or pure electrical vehicle presents electrical storage. Supercapacitors are nowadays the only available technology, which can provide great specific power (over $1 \mathrm{~kW} / \mathrm{kg}$ ) and great number of cycles at reasonable price, and safe and reliable work. Supercapacitors have other characteristics that make them attractive in hybrid vehicles, such as possibility of complete energy using (so-called regenerative breaking) for increasing energy efficiency, with no additional maintenance, great recovery of electrical energy, little toxicity and easy disposal after usage.

\section{Experimental}

\subsection{Construction}

The EDLC consists of two electrodes that are immersed in an electrolyte with a separator between them. The electrode consists of a current collector in contact with the active material. In EDLCs, the energy storage arises mainly from the separation of electronic and ionic charges at the interface between high-area electrode material and the electrolyte solution. In the EDLC, this charge separation distance, $d$, is reduced to the Helmholtz double-layer $d_{\mathrm{D}}$ thickness, which is defined as half the diameter of the adsorbed solvated ions at the electrode/solution interface [6].
A pilot supercapacitor has been made using commercially available activated carbon (AC) material, composite $\mathrm{RuO}_{2}+\mathrm{AC}$ and natural copper sulfide minerals as the active electrode material. Inactive part is watered by araldite mass. The connection of the mineral with current supplier is achieved with conducting silver glue. A supercapacitor prototype was made according to the sketch in Fig. 2. Nafion ${ }^{\circledR}$ Membrane N 115 (5 nm thick) (Ion Power, Inc.) played the role of separator, and it was soaked with a chosen electrolyte.

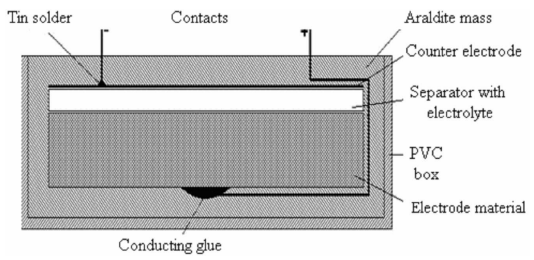

Fig. 2. Construction of the supercapacitor prototype.

\subsubsection{Carbon}

The electrode material should be electrochemically inert in the working potential, which is limited by the decomposition potential of the electrolyte.

Carbon material, such as carbon nanotubes and AC are very attractive as electrode materials in the EDLCs due to their large specific surface area and low mass density [7-10]. It is well known that the double layer capacitance on a clean graphite surface is fixed at around $20 \mu \mathrm{F} \mathrm{cm}^{-2}$.

Multiwalled (MWNT) and single walled (SWNT) nanotubes are well adapted to allow the electrolyte accessibility because of their morphology, high specific surface area and good mechanical properties.

\subsubsection{Ruthenium oxide}

Ruthenium oxide, $\mathrm{RuO}_{2}$, is a well-known electrode material with excellent electrocatalytic features for many electrochemical processes. While the anhydrous, crystalline rutile $\mathrm{RuO}_{2}$ is a metallic conductor, hydrous ruthenium oxide exhibits mixed electron/proton conductivity, which makes it a promising candidate for the use in energy-storage devices, such as electrochemical supercapacitors and direct methanol fuel cells (DMFCs) [11].

Hydrous ruthenium oxide is of amorphous structure consisting of more or less ordered $\mathrm{RuO}_{6}$ octahedra, depending on water content and temperature treatment. The specific capacitance of this material was reported to be as high as $720 \mathrm{~F} \mathrm{~g} \mathrm{~g}^{-1}$ and it is strongly dependent on the degree of ordering and crystalline water content.

The capacitive performances of hydrous ruthenium oxide can be improved if its pseudocapacitance is combined with double layer capacitance of carbon blacks. This can be done by preparing the carbon-supported $\mathrm{RuO}_{x} \mathrm{H}_{y}$.

\subsubsection{Copper sulfide minerals}

Numerous results of electrochemical researches on copper sulfides (covellite, $\mathrm{CuS}$ and chalcocite, $\mathrm{Cu}_{2} \mathrm{~S}$ ) can be 
found in the literature, but non referring to the material as a potential electrode material for capacitors. Investigation of behaviour of copper sulfide minerals during their anodic polarization and modeling of these reactions were performed at Technical Faculty in Bor. It was established that equivalent electrical circuit must contain very high capacitances, indicating a possibility of using copper sulfide minerals for electrochemical supercapacitor electrodes [12].

\subsection{The system for electrochemical characterization}

Electrochemical investigation methods are widely used for characterization of different kinds of materials, as well as of the processes in systems where the electrochemical reactions take part. There is a series of well known methods, but some new methods from electrotechnical area have been introduced [13]. So, first of all it was given an overview of the standard electrochemical methods and parameters, beginning with potential measurement and simple methods, such as chronopotentiometry and chronoamperometry, till electrochemical impedance spectroscopy. The last named method is adapted for systems containing large capacitances that became actually with the appearance of electrochemical supercapacitors. New methods are the Dirac voltage excitation and the Dirac current excitation. Measurement system described here allows application of electrochemical methods, as follows: measuring open circuit potential, chronopotentiometry, chronoamperometry, galvanostatic method, potentiostatic method, the Dirac voltage excitation, galvanodynamic method, cyclic voltammetry and electrochemical impedance spectroscopy.

For signal generation and data acquisition it was developed a measuring and control system based on PC Pentium 4. Beside PC, hardware consists of ADDA converter and external interface for analog signals conditioning. ADDA conversion is performed using commercially available NI 6251 converter from National Instruments, $\mathrm{M}$ series high-speed multifunction data acquisition. They have an onboard NI-PGIA2 amplifier designed for fast settling times and high scanning rates, ensuring 16-bit accuracy even when measuring all channels at maximum speeds.

Measurement interface has been designed for the needs of the electrochemical investigations by controlled current or voltage excitation and response registration.

The software platform for predicted measurement methods was National Instruments LabVIEW 8.2 package, which is regarded as a high standard in the area of modern virtual instruments. In LabVIEW, one builds a user interface by using a set of tools and objects. The user interface is known as the front panel. As an example, the description, as well as front panel has been showed for the electrochemical impedance spectroscopy.

\subsubsection{Electrochemical impedance spectroscopy}

In this method, before any measurement was done, on the outlet there has been put the direct (DC) voltage to last long enough in order to achieve a stationary regime, and then the current measurement can be started as a response to a complex excitation (superimposed alternating voltage of an order of magnitude millivolts on DC level up to $10 \mathrm{~V}$ ). The assigned parameters are: open circuit potential, $E_{\mathrm{OC}}$, direct voltage, $E_{\mathrm{DC}}$, and time of the regime duration, $t_{\mathrm{o}}$.

After the DC regime expires, the initial WHILE loop starts, in which the current value of the alternate voltage of the assigned amplitude $E_{\mathrm{mAC}}$ and the calculated frequencies (block DAQmx Write), the channel (AOCH1) have been generated. The frequency is calculated in the external loop according to octaves, in relation to the assigned values starting from $f_{\min }$ to $f_{\max }$.

The lowest frequency can be $1 \mu \mathrm{Hz}$ necessary for systems with high capacitances. The generated alternating voltage is being superimposed with already installed DC voltage and they both make together the excitement of the electrochemical system.

The current response, which in the pseudo stationary regime has also got the sinusoidal shape with a DC component, is measured on the analog input channel, being averaged, converted into an array and led in a block Array Max\&Min. On the base of this block it is possible to calculate the average maximal value of the superimposed component of the voltage excitation so that the outcome is the module of system impedance which is memorized and graphically displayed as a function of the logarithm of the frequency - Bode's diagram of an impedance (Fig. 3).

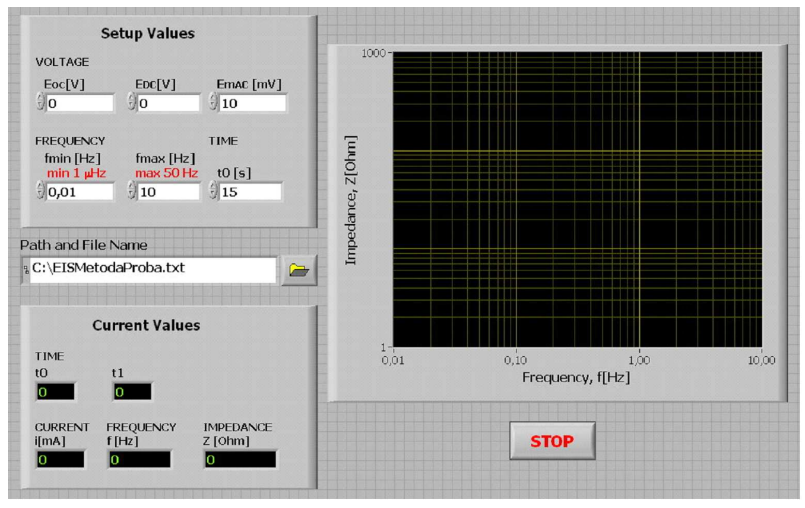

Fig. 3. Front panel for electrochemical impedance spectroscopy.

\section{Results and discussion}

\subsection{Carbon}

The starting material was activated carbon [Aktivkohle (zur Analyse), by MERCK], which was used as the base material for building the supercapacitor. In order to prepare supercapacitor electrodes, a mixture of activated carbon and the polymer binder - polyvinylidene difluoride (PVDF) - in weight ratio 10:1 was mixed in automatic mill for $30 \mathrm{~min}$. The given composite was then soaked with acetone in order to give a paste. 
A mixture with the activated carbon:PVDF (ratio 5:1) has been prepared, too. The mass of activated substance in each electrode was 160-200 mg. The electrodes were dried $24 \mathrm{~h}$ at room temperature and the dried electrodes were soaked in $3 \mathrm{M} \mathrm{H}_{2} \mathrm{SO}_{4}$ solution in vacuum vessel for 5 min at the presure of $5 \mathrm{kPa}$ and another 10 min under the presure of $72 \mathrm{kPa}$. The electrodes' capacitance was measured by making an EDLC cell. All the experiments were performed at room temperature.

Cycle voltammetry and galvanostatic charge/discharge measurements were used to investigate the capacitive performance of supercapacitors.

The results obtained with composite of the AC:PVDF ratio 5:1 showed that this system had large series resistance, so the other experiments were realized only with the samples of the AC:PVDF ratio 10:1. The active electrode in one of investigated EDLCs was made just with activated carbon material soaked in $3 \mathrm{M} \mathrm{H}_{2} \mathrm{SO}_{4}$ solution to form a paste.

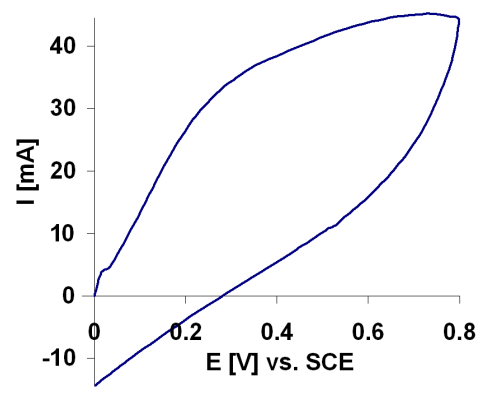

Fig. 4. Cyclic voltammogram (first cycle) of the capacitor built from carbon; the scan rate: $5 \mathrm{mV} / \mathrm{s}$.

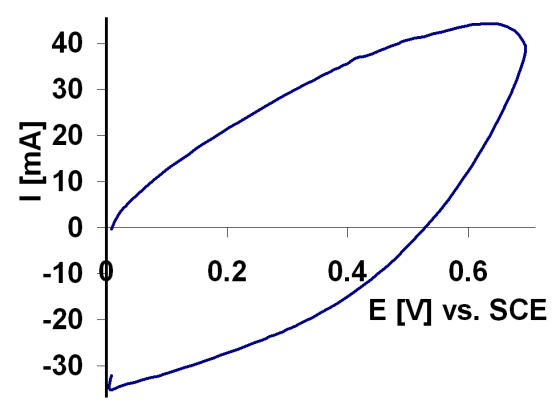

Fig. 5. Cyclic voltammogram (first cycle) of the capacitor built from carbon/PVDF mixture; the scan rate: $2 \mathrm{mV} / \mathrm{s}$.

Since the time constants of examined electrochemical systems were high, the standard cyclic voltammetry method at a low sweep rate was applied. A series of experiments was carried out on various electrochemical systems with varying sweep rate in order to determine the optimum working conditions. Figures 4 and 5 show the cyclic voltammograms obtained at scan rate of $5 \mathrm{mV} / \mathrm{s}$ and $2 \mathrm{mV} / \mathrm{s}$ for the capacitor built from activated car-

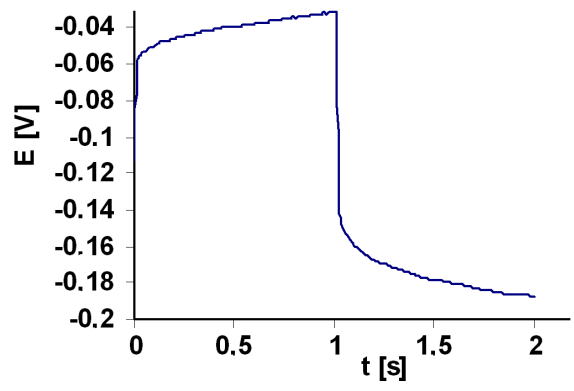

Fig. 6. Galvanostatic charge/discharge of the capacitor built from carbon; current of $\pm 10 \mathrm{~mA}$.

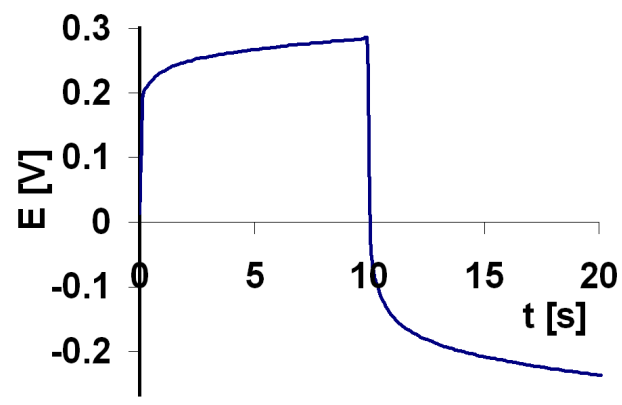

Fig. 7. Galvanostatic charge/discharge of the capacitor built from carbon/PVDF; current of $\pm 50 \mathrm{~mA}$.

bon and for the capacitor built from carbon/PVDF and mixture, respectively.

The shape of the curves presented in Figs. 4 and 5 suggests a nearly ideal behaviour of those types of capacitor. It is clear that the CV shapes were well defined with the large loop surface area. The specific capacitance for the capacitor presented in Fig. 4 was nearly $100 \mathrm{~F} / \mathrm{g}$, and for capacitor presented in Fig. 5, the capacitance was nearly $200 \mathrm{~F} / \mathrm{g}$.

The results of galvanostatic charge/discharge measurements are presented in Figs. 6 and 7 . Calculated resistance was about $4 \Omega$ for capacitor made by carbon

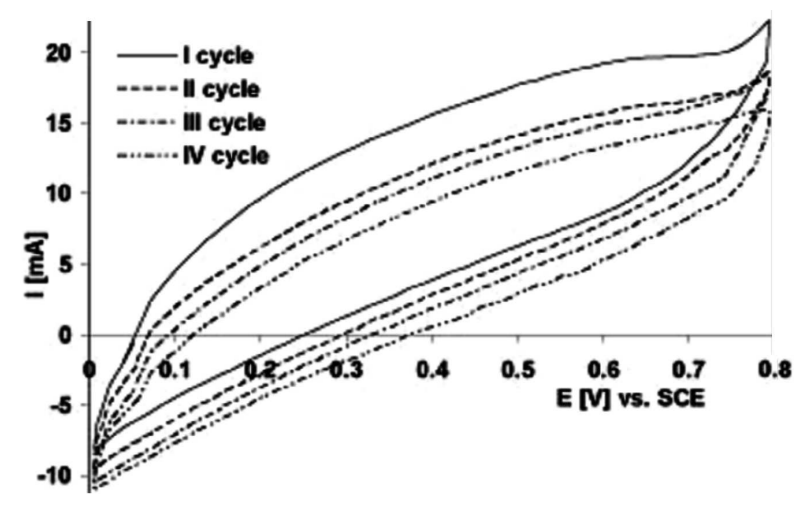

Fig. 8. Cyclic voltammograms of the capacitor built from carbon and Nafion 115 as a separator; scan rate of $5 \mathrm{mV} / \mathrm{s}$. 
(Fig. 6), and $3.5 \Omega$ for capacitor made by carbon/PVDF mixture (Fig. 7).

Cyclic voltammograms (four cycles obtained after galvanostatic investigations) of a capacitor built from carbon and Nafion 115 as separator are presented in Fig. 8 . It can be noticed that during the cycling, the values of current are lower in every next cycle.

\subsection{Composite $\mathrm{RuO}_{2}+\mathrm{AC}$}

Ruthenium oxide-based supercapacitors were first developed in the late 1970s by Conway in collaboration with Craig of Continental Group Inc. [14]. More recent work has been focused on the development of electrode structures and composites to take the best advantage of the high-specific capacitance of hydrous $\mathrm{Ru}$ oxide. In view of the dificulties in maintaining the specific capacitance of $\mathrm{Ru}$ oxide at high-electrode loading, there have been numerous studies of $\mathrm{Ru}$ oxide composites with various types of carbon [15]. Panić et al. [11] investigated the influence of the carbon black support on the composite capacitive properties comparing the data obtained with $\mathrm{BP}$ and $\mathrm{XC}$ carbons.

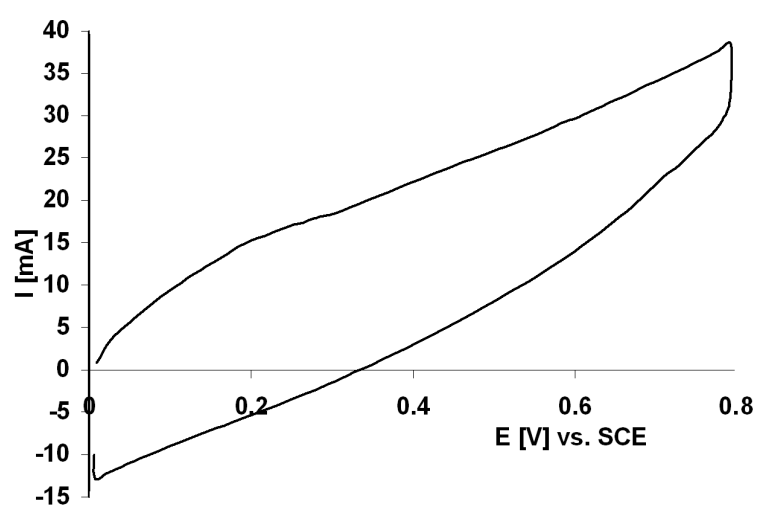

Fig. 9. Cyclic voltammograms of the capacitor built from $\left(\mathrm{RuO}_{2}+\mathrm{AC}\right) / \mathrm{PVDF}$ mixture; scan rate of $5 \mathrm{mV} / \mathrm{s}$.

The method of electrodes preparation was the same as in the case of $\mathrm{AC} / \mathrm{PVDF}$ mixture, but the active material was the composite $\mathrm{RuO}_{2}+\mathrm{AC}$ and the composite: $\mathrm{PVDF}$ ratio 10:1. The cyclic voltammogram obtained at scan rate of $5 \mathrm{mV} / \mathrm{s}$ is presented in Fig. 9. Calculated capacitance is about $50 \mathrm{~F} / \mathrm{g}$ and the system shows better cycleability than other materials.

\subsection{Copper sulfide minerals}

The next material were the samples of natural copper minerals covellite and chalcocite from Bor copper mine and "white-matte" — the inter-product in copper production, chemically similar chalcocite. Having performed a series of experiments it was found out that chalcocite showed the best features as a potential electrode material for supercapacitors, subsequently, it was thoroughly explored. From the examination by the EIS method it was obtained a capacitance of $29.9 \mathrm{~F}$ for chalcocite in $1 \mathrm{M} \mathrm{H}_{2} \mathrm{SO}_{4}+0.1 \mathrm{M} \mathrm{CuSO}_{4}$ and $65.8 \mathrm{~F}$ in $1 \mathrm{M} \mathrm{H}_{2} \mathrm{SO}_{4}+$ $0.5 \mathrm{M} \mathrm{CuSO}_{4}[7]$.

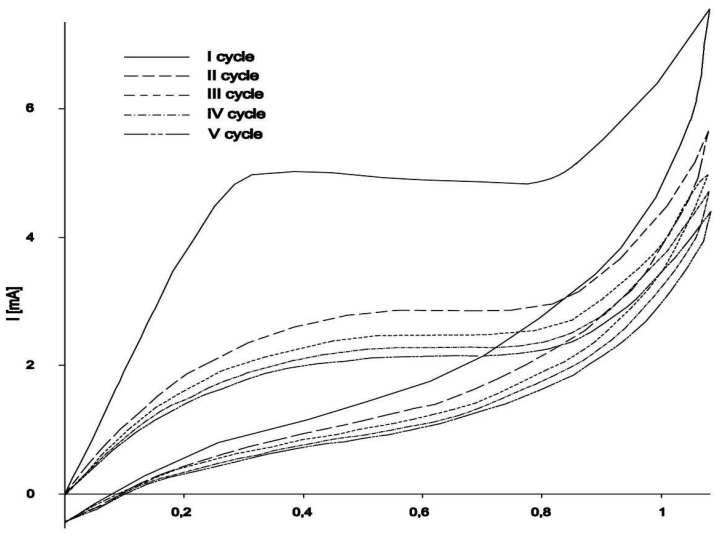

Fig. 10. Cyclic voltammograms of covellite in $1 \mathrm{M}$ $\mathrm{H}_{2} \mathrm{SO}_{4}+0.05 \mathrm{M} \mathrm{CuSO}_{4}+0.05 \mathrm{M} \mathrm{Na}_{2} \mathrm{SO}_{4}$; scan rate of $500 \mathrm{mV} \mathrm{s}^{-1}$.

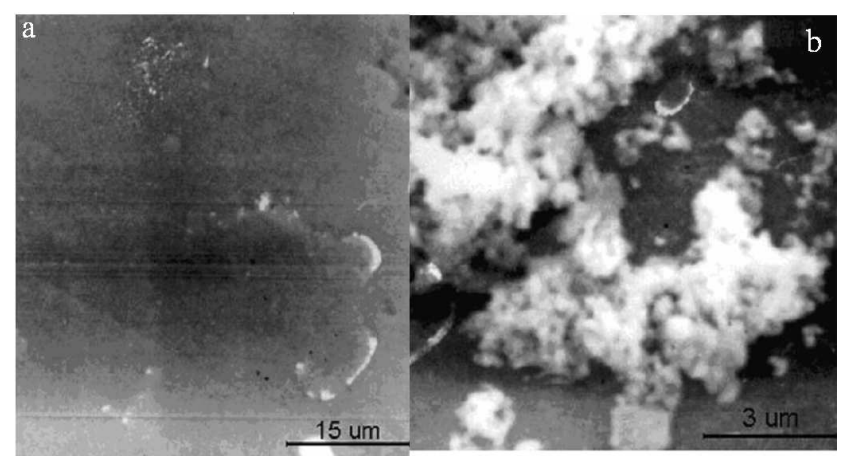

Fig. 11. Electronic microscopy pictures of the covellite sample before (a) and after (b) the application of galvanostatic impulse of $0.5 \mathrm{~mA}$ for the duration of $80 \mathrm{~s}$ in $1 \mathrm{M} \mathrm{H}_{2} \mathrm{SO}_{4}$ electrolyte.

The covellite electrode was examined in various electrolytes but the majority of experiments were done in aqueous solutions of sulfuric acid varying in concentration, with or without the addition of copper sulfate. Figure 10 presents a series of voltammograms obtained in $1 \mathrm{M} \mathrm{H}_{2} \mathrm{SO}_{4}+0.05 \mathrm{M} \mathrm{CuSO}_{4}+0.05 \mathrm{M} \mathrm{Na}_{2} \mathrm{SO}_{4}$ solution by cycling with the sweep rate of $500 \mathrm{mV} \mathrm{s}^{-1}$. It can be seen that the voltammetric current shows a steady decrease with the increase of cycle numbers. The existence of a compact solid reaction product remaining on the electrode surface may be considered as the main reason of that decrease. That product causes a slow diffusion in solid state, which leads to the increase of serial resistance and controls further reaction. Capacitance calculated from the first loop surface area is around $0.034 \mathrm{~F} \mathrm{~cm}^{-2}$ (electrode active surface area is $0.18 \mathrm{~cm}^{-2}$ ).

The polished surface of the material was analyzed by electronic microscopy before (Fig. 11a) and after 
(Fig. 11b) the application of galvanostatic impulse of $0.5 \mathrm{~mA}$ for the duration of $80 \mathrm{~s}$ in $1 \mathrm{M} \mathrm{H}_{2} \mathrm{SO}_{4}$ electrolyte.

Uniform surface of the non-treated sample confirms its high purity concerning natural mineral; natural minerals usually contain some impurities like quartz or pyrite. Some product appeared on the treated electrode surface during the anodic process. The structure of this product may be considered as the main reason of such high capacitance found out at covellite and other copper minerals.

\section{Conclusions}

The EDLCs are a new type of capacitors offering new features intermediate between those of batteries and conventional capacitors. Characteristics, applications, construction and system for electrochemical characterisation are described in the paper.

Presented results are obtained with the electrodes made of commercially available $\mathrm{AC}$ material, $\mathrm{RuO}_{2}+\mathrm{AC}$ composite and natural mineral covellite.

Characterization has been performed by an electrochemical system fully developed at Technical Faculty in Bor. The system is based on a PC P4, a commercially available ADDA converter and an external interface for analog signal processing.

Described results at this developed electrochemical capacitors are comparable with results in other laboratories in the world. PC based measurement system developed by authors is fully confirmed.

\section{Acknowledgments}

This work was financially supported by Ministry of Science and Technological Development of Republic of Serbia (Project No. 142048).

\section{References}

[1] H. Shi, Electrochim. Acta 41, 1633 (1996).

[2] K. Kimoshita, Carbon: Electrochemical and Physicochemical Properties, Wiley, New York 1988.

[3] T. Christen, M.W. Carlen, J. Pow. Sour. 91, 210 (2000).

[4] M. Endo, T. Takeda, Y.J. Kim, K. Koshiba, K. Ishii, Carbon Sci. 1, 117 (2001).

[5] H.L.F. Helmholtz, Ann. Phys. 89, 211 (1879).

[6] Ch. Emmenegger, Ph. Mauron, P. Sudan, P. Wenger, V. Hermann, R. Gallay, A. Zutte, J. Pow. Sour. 124, 321 (2003).

[7] M. Ue, Curr. Topics Electrochem. 7, 49 (2000).

[8] S. Iijima, Nature 354, 56 (1991).

[9] E. Frackowiak, F. Béguin, Carbon 39, 937 (2001).

[10] P. Bernier, W. Maser, C. Journet, A. Loiseau, M.L. de la Chapelle, S. Lefrant, R. Lee, J.E. Fischer, Carbon 36, 675 (1998).

[11] V. Panić, T. Vidaković, S. Gojković, A. Dekanski, S. Milonjić, B. Nikolić, Electrochim. Acta 48, 3805 (2003).

[12] Z. Stević, M. Rajčić-Vujasinović, J. Pow. Sour. 160, 1511 (2006).

[13] Z. Stević, Z. Andjelković, D. Antić, Sensors 8, 1819 (2008).

[14] B.E. Conway, J. Electrochem. Soc. 138, 1539 (1991).

[15] X. Liu, P.G. Pickup, J. Pow. Sour. 176, 410 (2008). 